NBER WORKING PAPER SERIES

\title{
A MODEL OF FORUM SHOPPING, WITH SPECIAL REFERENCE TO STANDARD SETTING ORGANIZATIONS
}

\author{
Josh Lerner \\ Jean Tirole \\ Working Paper 10664 \\ http://www.nber.org/papers/w10664
}
NATIONAL BUREAU OF ECONOMIC RESEARCH
1050 Massachusetts Avenue
Cambridge, MA 02138

August 2004

\begin{abstract}
We are grateful to a number of practitioners for helpful discussions and to Patrick Rey, Mike Rothschild, and Lucy White for comments. Emmanuel Fahri provided superb research assistance. In particular, he suggested and proved Propositions 13 and 14. We thank Harvard Business School's Division of Research and the U.S. National Science Foundation for financial support. All errors are our own. The views expressed herein are those of the author(s) and not necessarily those of the National Bureau of Economic Research.
\end{abstract}

(C2004 by Josh Lerner and Jean Tirole. All rights reserved. Short sections of text, not to exceed two paragraphs, may be quoted without explicit permission provided that full credit, including (C) notice, is given to the source. 
A Model of Forum Shopping, with Special Reference to Standard Setting Organizations

Josh Lerner and Jean Tirole

NBER Working Paper No. 10664

August 2004

JEL No. D71, D82, L15, O31

\section{ABSTRACT}

Owners of intellectual property or mere sponsors of an idea (e.g., authors, security issuers, sponsors of standards) often need to persuade potential buyers or adopters of the worth of their property or idea. To this purpose, they often resort to more or less independent certifiers. This paper analyzes the strategic choice of certifiers in rival and non-rival situations in a three-stage game. First, the owner/sponsor selects among potential certifiers. Certifiers differ in their degree of sympathy towards the owner/sponsor's interests relative to their concern for quality delivered to the users. Second, the certifier studies the offering and renders an opinion. The opinion consists of an endorsement (or lack thereof) and, possibly, some further demands for changes involving prices or offering characteristics. Third, the final users adopt or buy as a function of their perceived utility. In this context, the choice of certifier involves a basic trade-off: trying a tougher certifier reduces the probability of a positive opinion, but makes the users more likely to adopt the offering or willing to pay more for it in case of a positive opinion by the certifier. The paper first analyzes the sponsor's choices of certifier and design, as well as social preferences regarding these choices. More attractive standards lead to more friendly certification and fewer concessions to users. Regulation cannot improve on private choices in case of mildly attractive standards, and partial regulation reduces social welfare in case of attractive standards. Furthermore, the sponsor can costlessly delegate the design choice to the certifier when she can have her preferred choice of certifier, but must make more concessions to users than she would want to if the spectrum of certifiers is limited. The paper then extends the basic model to multiple categories of users, to the downstream presence of the sponsor, and to within-user-group network externalities. Finally, it studies strategic forum shopping by sponsors of competing standards.

Josh Lerner

Harvard Business School

Arthur Rock Center for Entrepreneurship, Room 214

Boston, MA 02163

and NBER

josh@hbs.edu
Jean Tirole

Institut d'Economie Industrielle

Manufacture de Tabacs

Bureau MF529 - Bat. F

21 allees de Brienne

31000 Toulouse FRANCE

tirole@cict.fr 


\section{Introduction}

Owners of tangible or intangible property or mere sponsors of an idea need to persuade potential buyers or adopters of the worth of their property or idea. To this purpose, they often resort to more or less independent certifiers. Rather than just publishing works on the web or in non-refereed books, academics typically submit their research to journals, which vary in how demanding their standards are and in their sympathy for various topics. Authors attempt to convince reputable publishing houses to print their work, and search for endorsements from well-regarded peers. Issuers of bonds or stocks are eager to receive certification from investment banks, analysts, and rating agencies, which differ in their reputation and independence. Technology sponsors attempt to build standards around their technologies by having them validated by standard setting organizations (SSOs) that range from fully independent to largely captive special interest groups (SIG).

This paper is a first pass at analyzing the strategic choice of certifiers in rival and non-rival situations. It employs a three-stage game. First, the owner / sponsor selects among potential certifiers. Certifiers differ in their degree of sympathy towards the owner / sponsor's interests relative to their concern for quality delivered to the users. Second, the certifier studies the offering and renders an opinion. The opinion consists of an endorsement (or lack thereof) and, possibly, some further demands for price changes or adjustments of the offering characteristics. Third, the final users adopt or buy as a function of their perceived utility.

In this context, the choice of certifier involves a basic trade-off: trying a tougher certifier reduces the probability of a positive opinion, but makes the users more likely to adopt the offering or willing to pay more for it in case of a positive opinion by the certifier.

Because we discuss some case study evidence from standard setting in the next section, we couch the framework as one in which the certifiers are SSOs. But as the examples provided above illustrate, the theoretical part of the paper has much broader applicability.

The paper is organized as follows. Section 2 provides some further motivation by dis- 
cussing sponsors' choice of SSOs. The theoretical part first analyzes the sponsor's choices of certifier and design, as well as social preferences regarding these choices (Sections 3 and 4). The paper then extends the basic model to multiple categories of users, to downstream presence of the sponsor, and to within-user-group network externalities (Section 5). Finally it studies strategic forum shopping by sponsors of competing standards (Section 6).

\section{Related literature}

\section{a) Literature on standards}

Despite the copious research on standards, it is striking how little work has addressed the question of how SSOs should be organized or how firms should choose between competing SSOs. Many of the papers in the literature focus only on de facto standard setting, where there is no role for a SSO in the model (e.g., Farrell and Saloner 1985). Alternatively, a number of works, both in economics and political science, have focused on settings where government bodies have played the key role in adjudicating between the desires of different parties about possible standards (e.g., Farrell and Shapiro 1992).

The economics literature analyzing SSOs has largely focused on just one role: that of a meeting room, i.e., a setting where the parties can negotiate. ${ }^{1}$ The other roles played by committees — such as facilitating information sharing, actively promoting design compromises, and certifying new standards - are ignored. And there is no need for more than one SSO in this setting, since the features of the SSO do not matter.

Farrell (1996) and Simcoe (2003) model the standard-setting process as a "war of attrition" between two parties with their own proposed standards. As in the rest of the literature, the various participants receive a larger share of the profits if their own standard is selected. Participants have private information about the quality of their proposed standard. These papers show that ultimately, the higher-quality technology will

\footnotetext{
${ }^{1}$ For example, in Farrell and Saloner (1988), two firms can choose between two incompatible technologies. They can do so by repeatedly talking with each other (seen as akin to a SSO), by product market competition (de facto standard setting in the marketplace), or a hybrid between the two approaches. The authors show that the committee process is more likely to arrive at a high-value consensus than product market competition, but that it usually takes longer. The hybrid approach is likely to dominate both of the alternatives.
} 
be selected, but that the delays will be a function of the presence of vested interests. In Simcoe's model, which incorporates explicit voting by SSO participants, relaxing the hurdle needed to approve a standard will reduce delay, but may also reduce the probability that the best technology is selected. Reducing vested interests (e.g., by adopting rules that limit the utilization of intellectual property used in standards) will reduce delays. When vested interests are already quite high, or when the dispersion of quality is high enough, decreasing vested interests will boost rather discourage the innovations undertaken by the two firms.

The nature of SSO organizations, and the impact of these structures on the standards selected, has attracted little empirical attention as well. Most of the empirical pieces have been based on individual case studies (e.g., Sirbu-Zwimpfer 1985) or comparisons of a small number of cases (Besen-Johnson 1985, Weiss-Sirbu 1990). They have largely focused on a question somewhat different from the one that we examine, namely the way in which an individual SSO chooses between competing standards that rival firms are championing.

\section{b) Literature on financial certification}

Many financing decisions are characterized by insufficient information held by potential investors, that may result in a breakdown of the market. A large literature in financial economics, beginning with Diamond (1984), has highlighted the importance of intermediaries in certifying firms seeking to raise external financing. ${ }^{2}$ Among the institutions whose certification role has been highlighted in theoretical and/or empirical studies have been commercial and investment banks, venture capitalists, large-block shareholders, analysts, auditors, and rating agencies. ${ }^{3}$ This literature has largely ignored the strategic choice of

\footnotetext{
${ }^{2}$ E.g., Admati-Pfleiderer (1994), Chemmanur-Fulghieri (1994), Lizzeri (1999), and Peyrache-Quesada (2003).

${ }^{3}$ These intermediaries can play this role for three reasons. First, the intermediaries are able to generate intensive knowledge about the issuing firm due to their involvement with the firm. Second, the intermediary must have acquired substantial reputational capital that it risks by certifying the firm. If the firm subsequently proves to have misstated its potential, then the intermediary's reputation capital may be tarnished. Third, the intermediary may take a financial stake in the firm.
} 
certifiers by firms (often assuming that the intermediary is a monopolist), as well as several other issues considered in this paper. A notable exception is Morrison-White (2004), in which banks apply to regulators with heterogenous abilities to screen. A successful application allows banks to raise more deposits, all the more so as the regulator's screening ability is perceived as high. The focus and analysis in Morrison and White are rather different from ours, as their primary interest lies in examining the impact of international financial liberalization in allowing banks to benefit from foreign supervision.

\section{c) Literature on real authority}

This paper is also related to the literature on real authority within organizations. Using the terminology of Aghion and Tirole (1997), in this paper, the sponsor selects a non-congruent SSO that will have real authority over the users.

Although quite different in motivation and execution, our work is therefore related to that of Dessein (2002). In his model, a principal delegates formal authority to a supervisor with preferences that are intermediate between hers and the agent's. This step motivates the agent to exercise initiative, because he knows that the supervisor will listen to him.

It is also related to Caillaud and Tirole's (1999) work on ideological bias. They show that a party with partisan rank-and-file members is, in the eyes of uninformed voters, a more credible certifier for the strength of the (optimally) centrist platform of its leadership than a party with a centrist rank-and-file. This relationship implies that partisan parties may defeat centrist ones even in a Downsian world, where the median voter dictates political choices.

\section{An illustration ${ }^{4}$}

One way to illustrate these issues is to consider standard-setting organizations. Our interviews suggest two patterns that are consistent with the premises of the theoretical

\footnotetext{
${ }^{4}$ This section is based on interviews with SSO participants and corporate managers responsible for standards.
} 
analysis below:

- that SSOs differ in terms of the extent to which they are attuned to user's desires, and in the way sponsors have control over the standard;

- that certification is a real concern of firms sponsoring new proposed standards.

The first point can be illustrated by the fact that a firm seeking to get a technology adopted as a new standard frequently faces a choice of three or four SSOs from which it could seek certification. In making a choice between existing SSOs, or between new and existing organizations, the prestige of the body is an important consideration. While for many firms, the ideal standard is a de facto one, which it or perhaps a special interest group promulgates and everyone else adheres to, in many cases it cannot get a standard established without being approved by a prestigious established SSO.

Relatedly, firms believe that SSOs differ considerably in the extent to which a sponsoring firm can influence the process. (The key variables include the composition of the membership and the rules employed in reviewing proposed standards.) SSOs also differ in the extent of requirements regarding the disclosure and licensing of intellectual property. ${ }^{5}$

To illustrate these general points, consider two SSOs with similar mandates. (Even more extreme differences would have been seen if we compared an SSO and a special interest group.) The Internet Engineering Task Force (IETF) is responsible for engineering and developing protocols for the Internet. It was formally established in 1986, but predecessor organizations date back at least as far as the early 1970s. The International Telecommunications Union (ITU) has been seeking to ensure the standardization of communications since a 1865 agreement to facilitate the interconnection of national telegraph systems. With a membership encompassing telecommunication policy-makers and regulators, network operators, equipment manufacturers, hardware and software developers,

\footnotetext{
${ }^{5}$ Firms' preferences differ considerably across technologies. In some cases, they may very strongly feel that it is important that they not commit to licensing their key patents; in other cases, they do not mind making such commitments.
} 
and regional standards-making organizations, today it seeks to develop standards and policies for all aspects of telecommunications.

These two groups differ along a number of dimensions. One aspect is the nature of the participants. At the IETF, individual members make decisions. At the ITU, national governments are the key voting bodies; that is, the governments typically have formal or informal bodies that seek to reconcile the divergent views of domestic firms and to form a consensus, and in many cases the largest firms have dominated these advisory bodies. ${ }^{6}$

A second difference is the degree to which corporations can influence the process. This reflects the way in which the two SSOs are organized, particularly the role that the area directors play. At the ITU, the area director is primarily responsible for the managing the standard-setting process. At the IETF, the area director has responsibility for both the technical quality and process. This often leads to a more activist approach. As a result, efforts by corporations to shape the standard-setting agenda were often less successful. This point can be illustrated by considering the process of initiating new projects at the ITU and IETF. At the ITU, a proposal for a new project is brought to a study group. After reaching a consensus, the work begins while formal approval is solicited. The proposed research agenda is circulated to the membership at large, an advisory group, and the body of chairs of the various working groups. While all parties are free to suggest changes, the study group is relatively free to accept or reject the proposed modifications. At the IETF, however, the area (an equivalent of a study group) must begin with a written proposal to their area director before initiating a project. The area director will discuss the proposal with his peers, and then commence a formal evaluation procedure that entails holding one or two meetings of prospective contributors. The area director will assess both the number of attendees and the quality of the discussion at the meetings before giving permission

\footnotetext{
${ }^{6}$ It should be noted that while in its first decades, engineers largely did participate in the IETF as objective individual experts, regardless of their corporate affiliations, and the agendas of these firms and decisions were largely driven by technological considerations, today this has changed somewhat. The much greater stakes associated with the adoption of particular standards (particularly in light of the bleak commercial environment for Internet and communications firms) implies that pressure is put on engineers in some cases to conform to corporate priorities during the decision-making process.
} 
whether to proceed with the project or not. While there is an appeal process, the area director's decision typically is the last word. As a result of the difficulty that individual firms frequently encounter in shaping the IETF agenda, it has been argued that the ITU is a much more sympathetic venue for proposals by large telephone companies.

\section{Forum shopping: basics}

\subsection{Model}

We now present a simple model of sponsor's choice among SSOs. In subsequent sections, we introduce some refinements.

Sponsors and users: Consider the sponsor (or group of sponsors) of a potential standard. The standard, if adopted, will yield profit $\pi>0$ to the sponsor, and utility $U$ for the users of the standard. For the moment, we distinguish between sponsor and users; later on, the sponsor will also have a downstream presence. Also, users should be understood in a broad sense, and include in particular future innovators building on the technology. Users adopt the standard only if they perceive $U$ to be positive.

SSOs: The sponsor chooses an SSO. SSOs are characterized by their user-friendliness, or more generally, by the ease with which a sponsor can get his standard approved by the SSO. A SSO has objective function

$$
U+\alpha \pi
$$

For analytical convenience, we will assume in much of the paper that there is "free entry in the SSO market": there is a continuum of SSOs indexed by $\alpha \in[0, \infty)$. Free entry in the SSO market is a strong assumption, but it allows us to use first-order conditions to capture our main points in a very simple manner.

The parameter $\alpha$ (which is common knowledge for each SSO) measures the conflict of interest. It may be very large for a SIG, and very low for an SSO with a user orientation. In general, $\alpha$ reflects, among other factors:

- the ease with which a yes vote can be obtained: simple majority rule, absence of a 
requirement of multiple interoperable implementations, and so forth,

- the composition of the board (users versus members of SIG),

- whether the users are direct or indirect users (downstream firms that compete with each other may pass through some of their cost increases - or, in Section 4, an increase in the royalty rate - to the final consumer, and therefore their stake may be smaller than that of users as a whole),

- the SSO's reputational concern vis-à-vis users.

Users' utility: Users have utility

$$
U=a+b
$$

where $a$ is common knowledge and serves to calibrate the initial strength of the proposed standard. This strength depends on common-knowledge technical merits and on the existence of alternative technologies. In particular, $a$ is smaller in the presence of an alternative technology, since the users' utility must be taken net of the opportunity cost of adopting this particular technology.

The parameter $b$ is unknown to both sponsor and users and reflects the unobserved quality of the proposed standard for the users: How promising is the technology itself? Is the technology likely to be blocked by (valid) patents? Or, if the technology is composed of multiple pieces of intellectual property owned by several sponsors, how well do those pieces interact with each other to benefit the users? Allowing for some private information about $b$ held by the sponsor would complicate the analysis but not reverse our main conclusions, as discussed below. Note also that SSOs all observe the same $b$. Thus, the sponsor, if she uses multiple SSOs, will not do so because she wants a second opinion after a first negative one. ${ }^{7}$

Let $F(b)$ denote the cumulative distribution function. We assume that $F$ has the standard monotone hazard rate property: $f /[1-F]$ is increasing. This property in turn

\footnotetext{
${ }^{7}$ Morrison and White (2004) develop a model in which certifiers make mistakes and economic agents looking for certification may sequentially go down the list of certifiers. Meyer and Rothschild (2004) adds incomplete information to the standard matching model, and apply this to sequential acceptation processes.
} 
implies that

$$
m(b) \equiv E[\widetilde{b} \mid \widetilde{b} \geq b]
$$

grows with $b$ at a rate lower than 1 (e.g., An [1998]). Furthermore:

$$
m^{\prime}(b) \equiv\left[\frac{f(b)}{1-F(b)}\right][m(b)-b] .
$$

\section{Timing}

We consider the following three-stage game:

(1) The sponsor chooses an SSO, that is $\alpha$.

(2) The SSO learns $b$ (more generally, it could learn a signal of $b$ ), and then chooses whether to recommend the standard.

(3) Users decide whether to adopt the standard.

We believe these assumptions about timing are plausible. SSOs do indeed frequently involve end users, and consequently can get a sense of a proposed standard's potential value. Because many technologies are still in their early stages at the time that the standard is endorsed, users frequently defer the decision whether to employ the standard until after it has been endorsed. In this bare-bones version of the model, the SSO plays a pure certification role. In practice, besides this central function, SSOs play the auxiliary roles of setting constraints on sponsors (e.g., disclosure and licensing requirements) and of inducing a convergence of rival sponsors on a unique standard. Such roles, which are discussed in the next sections, are less fundamental in that the sponsor(s), unlike for the certification function, can achieve the outcomes by themselves.

\subsection{Equilibrium forum shopping}

At stage (2), the SSO approves the standard when facing signal $b$ if and only if ${ }^{8}$

$$
[a+b]+\alpha \pi \geq 0 \text {. }
$$

\footnotetext{
${ }^{8}$ We ignore the "babbling equilibria" in which the users never adopt the standard and the SSO approves randomly (this equilibrium exists if $a+E[b]<0$ ) or else the SSO always recommends and users adopt regardless of the recommendation (this equilibrium exists if $a+E[b]>0$ ). Given this, the continuation equilibrium outcome for a given $\alpha$ is unique.
} 
A higher $\alpha$ means a more likely approval.

Users adopt the standard when approved by the SSO if and only if ${ }^{9}$

$$
E[a+b \mid a+b+\alpha \pi \geq 0] \geq 0
$$

or

$$
a+E[b \mid b \geq-(a+\alpha \pi)] \geq 0 .
$$

The left-hand side of inequality (1) decreases with $\alpha$ and increases with $a$.

The sponsor chooses the highest possible $\alpha$ subject to the credibility constraint (1).

Hence:

- If $a+E[b] \geq 0$, then $\alpha^{*}=\infty$ : the technology will be adopted as a de facto standard anyway, and so there is no point risking being turned down by a standard setting organization.

- If $a+E[b]<0$ (the interesting case), then

$$
a+E\left[b \mid b \geq-\left(a+\alpha^{*} \pi\right)\right]=0
$$

or

$$
a+m\left(b^{*}\right)=0
$$

where

$$
b^{*} \equiv-\left(a+\alpha^{*} \pi\right)
$$

Because the conditional mean $m(\cdot)$ has slope between 0 and 1 , equation (2) defines a single and increasing solution $\alpha^{*}(a)$.

Note also that the sponsor has no incentive to apply to a more sympathetic SSO $\left(\alpha>\alpha^{*}\right)$ when turned down, as the standard will then not be adopted anyway:

$$
a+E\left[b \mid-\left(a+\alpha^{*} \pi\right)>b \geq-(a+\alpha \pi)\right]<0 .
$$

\footnotetext{
${ }^{9}$ In order to avoid "openness problems," we will assume that users adopt with probability one if (1) is satisfied with equality (this is indeed the only equilibrium possibility).
} 
Proposition 1 The weaker the proposed standard, the more credible the SSO selected by the sponsor: $\alpha^{*}$ increases with a. The sponsor's expected payoff is then

$$
\left[1-F\left(-a-\alpha^{*}(a) \pi\right)\right] \pi
$$

In the conversations we had with firms active in the standard-setting process, this proposition was repeatedly corroborated. Our interviewees emphasized that they only selected as strong a SSO as was needed to gain widespread acceptance of the standard.

We may compare the allocation resulting from the sponsor's choice of SSO and that that would obtain under a social planner putting an exogenous relative weight $w$ on the sponsor's interests relative to the users'. Such a social planner would want adoption whenever $b \geq \widehat{b}$, where

$$
a+\widehat{b}+w \pi=0
$$

For attractive standards $\left(\alpha^{*}(a)>w\right)$, the sponsor chooses an SSO that is too friendly from a social viewpoint, and so the social planner would like to impose a less complacent SSO.

For less attractive standards $\left(\alpha^{*}(a)<w\right)$, by contrast, the social planner finds users to be too reluctant to adopt the technology (as they do not internalize the sponsor's benefit). Yet there is nothing that the social planner can do. An SSO that was more friendly than $\alpha^{*}(a)$ would be unable to get users on board by endorsing the standard.

Proposition 2 From the point of view of a social planner putting relative weight $w$ on the sponsor's interests relative to the users', the sponsor's choice of SSO is

- too complacent if the technology is very attractive $\left(\alpha^{*}(a)>w\right)$,

- constrained optimal if the technology is less attractive $\left(\alpha^{*}(a) \leq w\right)$.

\subsection{Can the sponsor do better?}

One may wonder whether the strategy described in Section 3.2 is overall optimal for the sponsor. We have indeed assumed that the sponsor applies to a single SSO; the sponsor 
could apply to multiple SSOs either simultaneously or sequentially. Also, the SSO issues a 0/1 recommendation; the SSO could convey more information (for example, announce b). It turns out that, in our model, the sponsor cannot deviate from the simple strategy analyzed thus far and obtain a higher payoff:

Proposition 3 The sponsor cannot achieve an expected payoff higher than the one (defined in Proposition 1) that she obtains by applying to a single SSO with characteristic $\alpha^{*}(a)$ and issuing a yes/no recommendation.

Proof: For any extensive form of the standardization game ${ }^{10}$ and for any $b$, let $X(b) \in[0,1]$ denote the present discounted probability of adoption by the users when the state of nature is $b .^{11}$

An upper bound on the sponsor's payoff is

$$
\begin{aligned}
& \max _{\{X(\cdot)\}} E[X(b) \pi] \\
& \text { s.t. } \\
& E[X(b)(a+b)] \geq 0 \\
& \text { and } \\
& 0 \leq X(b) \leq 1 \text { for all } b .
\end{aligned}
$$

The first constraint comes from the users' individual rationality: Were they to expect a negative utility, then they might as well never adopt. Letting $\beta$ denote the shadow price of this constraint, the first-order condition is:

$$
\begin{aligned}
X(b)=1 & \text { if } \pi+\beta(a+b)>0 \\
=0 & \text { if } \pi+\beta(a+b)<0 .
\end{aligned}
$$

\footnotetext{
${ }^{10}$ For example, the sponsor could apply to an arbitrary number $n_{0}$ of SSOs of her choice at date 0 , who would then issue recommendations, resulting in either user adoption, or in non-adoption, in which case at date 1 the sponsor could apply to an arbitrary number $n_{1}$ of SSOs, and so forth.

${ }^{11}$ For example, if $\delta$ is the discount factor and $x_{t}(b)$ denotes the equilibrium unconditional probability that the standard is adopted by the users when $b$ obtains $\left(0 \leq \Sigma_{0}^{\infty} x_{t}(b) \leq 1\right)$, then

$$
X(b)=\Sigma_{0}^{\infty} \delta^{t} x_{t}(b) .
$$
}


The corresponding allocation is implemented through the choice of an SSO with characteristic:

$$
\alpha=\frac{1}{\beta}
$$

Our interviewees indeed expressed skepticism about approaching a number of SSOs simultaneously. It is unclear that a second SSO's endorsement necessarily leads to enough additional certification to make it worth the trouble. ${ }^{12}$

Obviously, we do not want to imply that sponsors will always apply to a single SSO. Indeed, the sponsor in this model might want to apply to multiple SSOs if the latter observed only noisy measures of users' utility. Furthermore, Section 5.2 and Section 6 will demonstrate that applying to multiple SSOs may benefit a sponsor when either users are heterogeneous or there is competition from another technology. ${ }^{13}$ Proposition 3 , however, identifies a robust factor encouraging sponsors to focus on a single SSO: There is no point being more ambitious than needed for adoption, as the stigma from rejection by a tough SSO undoes the beneficial impact of endorsement by a more friendly one.

\subsection{Sponsor has private information about user benefit}

We have assumed that the sponsor has no information on the SSO's future assessment $b$ of user benefit. Suppose by way of contrast that the sponsor receives a signal $\sigma$ about $b$ with joint distribution $H(\sigma, b)$ and conditional distribution $G(b \mid \sigma)$.

Proposition 4 When the sponsor receives a private signal $\sigma$ regarding the SSO's assessment variable $b$ :

(i) it is still an equilibrium for the sponsor to choose SSO with preference $\alpha^{*}(a)$, regardless of her information $\sigma$ about the SSO's later assessment of the standard's user benefit;

\footnotetext{
${ }^{12}$ Another reason raised by interviewees is related to the concessions that are made by the sponsors: They asserted that it is almost inevitable that multiple standards will emerge: each group is likely to have its own approach, which will lead to them making different choices.

${ }^{13} \mathrm{~A}$ third reason why a sponsor may apply to multiple SSOs is that the latter may make mistakes: see Morrison-White (2004).
} 
(ii) more generally, the equilibrium choice of SSO does not reveal anything about the sponsor's information.

Proof: For a given $\alpha$, it is still the case that the SSO endorses the standard if and only if

$$
a+b+\alpha \pi \geq 0
$$

defining a threshold $\widehat{b}(\alpha)$. Let $F_{\alpha}(b)$ denote the users' updated beliefs about $b$ given that the sponsor chooses $\mathrm{SSO} \alpha$ (users update their beliefs about $\sigma$ and thereby about $b$ ). Let $m_{\alpha}(b) \equiv E_{\alpha}(\widetilde{b} \mid \widetilde{b} \geq b)$ denote the corresponding conditional mean. Consider then the set of $\alpha$ s that lead to adoption:

$$
\left\{\alpha \mid a+m_{\alpha}(\widehat{b}(\alpha)) \geq 0\right\}
$$

The sponsor solves:

$$
\max _{\left\{\alpha \mid a+m_{\alpha}(\widehat{b}(\alpha)) \geq 0\right\}}\{[1-G(\widehat{b}(\alpha) \mid \sigma)] \pi\} .
$$

The sponsor, regardless of her information $\sigma$, is better off choosing the highest $\alpha$ in this set so as to maximize the probability of endorsement. The highest point in the set may be equal to or strictly smaller than $\alpha^{*}(a)$, but there must be full pooling in equilibrium. The equilibrium in which all types $\sigma$ select $\operatorname{SSO} \alpha^{*}(a)$ is Pareto optimal from the point of view of the sponsor's types.

Remark: This absence of signaling through the choice of SSO does not carry over to the case where the sponsor is present in the downstream market (see Section 5.1).

\subsection{Scaleability}

Top scientific articles are often submitted to top-rank journals. One reason for this is that these journals have a wider circulation. But the main reason is the authors' benefit from 
publication $(\pi)$ is increasing is the quality $(s)$ of the journal (indeed, in the Internet era, the circulation argument has become less important and the certification is tantamount). ${ }^{14}$

A simple variation of our model provides a good account of this behavior: Suppose that a journal of quality $s$ decides to publish the paper if and only if:

$$
[a-\sigma(s)]+b \geq 0
$$

where $a$ is the paper's observable quality (known by the author), $\sigma(s)$ the journal's "standard" $\left(\sigma^{\prime}(s)>0\right)$ and $b$ the (referees' and editors') personal assessment variable. We leave aside for the moment any preference for the topic or author (the $\alpha$ term). The author's objective function is:

$$
[1-F(\sigma(s)-a)] \pi(s)
$$

The cross-partial derivative of the logarithm of this utility function wih respect to $a$ and $s$ is positive, and so, by revealed preference, a higher-quality paper $(a)$ is submitted to a better journal $(s)$. [This result generalizes to the introduction of a journal preference for topics or authors as long as it is not too strong (i.e., $\sigma^{\prime}(s)-\alpha \pi^{\prime}(s)>0$ ). Solving for the equilibrium behavior in presence of such preferences is an interesting topic for research.]

\section{Standard design}

Section 3 assumed that the characteristics of the potential standard are fixed. In practice, the sponsors can choose, and the SSO can try to alter, various characteristics of the proposed standard. These may include which features are included, whether patents or applications are disclosed, the terms of licensing (whether or not the sponsor must commit to license its patents on a reasonable and non-discriminatory (RAND) or a royalty-free basis), the exclusion of patented material requiring a more elaborate implementation,

\footnotetext{
${ }^{14}$ More generally, our basic model understates the importance of scaleability. Scaleability plays an important role in Morrison-White (2004), in which an endorsement by a more able regulator allows a bank to attract a higher volume of deposits.
} 
and so forth. The design question arises in other applications of the theory as well; for example, authors can offer, or editors of the journal demand, a shorter paper, a different emphasis, less ambitious claims, or broader citations.

\subsection{Strategic design by the sponsor}

To reflect this discussion, let us introduce an endogenous parameter $c$ of "concessions" to the users. ${ }^{15}$ The users' utility is now.

$$
U=a+b+c
$$

A higher $c$ may stand for a modification of the standard favorable to the users or for a commitment to a lower licensing price. Concessions are costly to the sponsor: the profit is now $\pi(c)$, with $\pi^{\prime}<0$ and $\pi^{\prime \prime} \leq 0$. We assume for the moment that the sponsor selects both $\alpha$ and $c$.

Following the lines of the previous analysis, the standard is adopted by the users following an endorsement by the SSO if and only if:

$$
a+c+E[b \mid b \geq-(a+c+\alpha \pi(c))] \geq 0 .
$$

The sponsor then solves

$$
\max _{\{\alpha, c\}}[1-F(-(a+c+\alpha \pi(c)))] \pi(c)
$$

subject to (3). Condition (3), satisfied with equality, defines $\alpha$ for a given choice of $c$ :

$$
b^{*} \equiv-[a+c+\alpha \pi(c)]=m^{-1}(-(a+c)) .
$$

Thus, the maximization can be rewritten as:

$$
\max _{\{c\}}\left[1-F\left(m^{-1}(-(a+c))\right)\right] \pi(c),
$$

\footnotetext{
${ }^{15}$ For simplicity, we assume that the sponsor chooses $c$. Sponsors actually sometimes employ a sequential strategy. In these cases, they recruit a close-in set of peers into a special interest group, where the real technological choices (and therefore the choice of $c$ ) are made, and then approach a larger SSO to get the standard ratified.
} 
or

$$
\max _{\{c\}}\left\{\log \left[1-F\left(m^{-1}(-(a+c))\right)\right]+\log \pi(c)\right\} .
$$

This latter objective function is concave. Taking its derivative and using the expression of $m^{\prime}$ :

$$
\frac{1}{m\left(b^{*}\right)-b^{*}}+\frac{\pi^{\prime}(c)}{\pi(c)}=0
$$

where

$$
b^{*} \equiv m^{-1}(-(a+c))
$$

Furthermore, equation (4), together with the identities $a+c+m\left(b^{*}\right)=0$ and $a+b^{*}+$ $c+\alpha \pi=0$, yields:

$$
1+\alpha \pi^{\prime}(c)=0
$$

Proposition 5 (i) The equilibrium concession is given by

$$
\frac{1}{m\left(b^{*}\right)-b^{*}}=\frac{-\pi^{\prime}(c)}{\pi(c)}, \text { where } b^{*}=m^{-1}(-(a+c)) .
$$

(ii) The weaker the proposed standard, the more extensive the concessions and the more credible the SSO selected by the sponsor: $c^{*}$ decreases with a and $\alpha^{*}$ increases with a.

Proof: Part (i) has already been demonstrated. Part (ii) is obtained by differentiating (4).

We can further extend Proposition 3. One might think that state-contingent concessions may be desirable. This is, however, not the case:

Proposition 6 The sponsor cannot achieve an expected payoff higher than the one (defined in Proposition 5) that she obtains by fixing c, and applying to a single SSO issuing a yes/no recommendation. 
Proof: The proof follows closely that of Proposition 3. Let $x_{t}(b)$ denote the present discounted probability of adoption at $t$, and $c_{t}(b)$ denote the state-contingent concession, with $\pi\left(c_{t}(b)\right)$ denoting the corresponding profit. ${ }^{16}$

The upper bound on the sponsor's profit is given by

$$
\begin{aligned}
& \max _{\left\{x_{t}(\cdot), c_{t}(\cdot)\right\}} E\left[\Sigma_{0}^{\infty} x_{t}(b) \pi\left(c_{t}(b)\right)\right] \\
& \text { s.t. } \\
& E\left[\Sigma_{0}^{\infty} x_{t}(b)\left[a+b+c_{t}(b)\right]\right] \geq 0
\end{aligned}
$$

and

$$
0 \leq \Sigma_{0}^{\infty} x_{t}(b) \leq 1 \text { for all } b .
$$

Letting $\beta$ denote the shadow price of the first constraint, one obtains a constant concession $c:$

$$
\pi^{\prime}(c)+\beta=0
$$

and

$$
x_{0}(b)=1 \text { if } \pi(c)+\beta(a+b+c) \geq 0 ; x_{t}(b)=0 \text { otherwise. }
$$

This policy is again implemented by an SSO with type $\alpha=1 / \beta$.

Before applying Proposition 5 to licensing fees, we may ask two further questions related to the timing of concessions:

- We have assumed that the sponsor defines the standard's specification $(c)$ and then submits it to the SSO. This is a simplification. Certainly in many cases, the determination of the level of concessions is in the hands of the SSO or is determined through a negotiation of the two parties. Is the final outcome different if the sponsor applies to a SSO, which then picks the standard's specification and decides whether to approve it? That is, is there a cost for the sponsor of deferring to the SSO?

- Another issue arises if the specification $c$ cannot be set at the standard setting date and will be at the discretion of the sponsor after the SSO has made a recommendation but

\footnotetext{
${ }^{16}$ In principle, $c_{t}(b)$ could be a random variable. Because of the concavity of $\pi$, though, we are characterizing the upper bound on the sponsor's profit.
} 
before the users have adopted the standard. That is, is there a cost for the sponsor of not being able to commit to a specification?

The answer to these two questions turns out to be negative under free entry into the SSO market:

- Deferrence to the SSO.

Suppose that the sponsor does not select $c$, and leaves it to the discretion of the SSO. If the latter has type $\alpha$, it chooses the extent of the concessions $c$, given by

$$
c^{*}(\alpha)=\arg \max \{c+\alpha \pi(c)\} .
$$

$c^{*}(\alpha)$ is a decreasing function of $\alpha$.

The sponsor cannot do better by deferring to the SSO since she can always duplicate the SSO's choice of specifications. But she might perhaps do better by not deferring since she has two degrees of freedom ( $\alpha$ and $c$ ) instead of one $(\alpha)$. For example, it might, in order to persuade users, have to choose a tough SSO, which would then impose substantial concessions. That this is actually not a concern results from equation (5), which shows that the optimal concession can be delegated to the SSO.

Proposition 7 Under free entry for SSOs, the sponsor can costlessly defer the choice of specification c to the selected $S S O$.

\section{- Non-commitment.}

Suppose now that the sponsor cannot commit in advance on the terms that will be offered to users. We thus assume that the sponsor sets $c$ after the SSO has rendered an opinion, but before the users choose whether to adopt the sponsor's technology (for example, $c$ may relate to the choice of licensing fee, as explored below). The SSO must then take a forward-looking perspective and anticipate the specification that will be selected by the sponsor. 
Thus, if the equilibrium threshold for the SSO is $b^{*}$, and assuming that the SSO has endorsed the technology, the sponsor selects the specification $c^{*}$ so as to extract the users' surplus:

$$
a+m\left(b^{*}\right)+c^{*}=0 .
$$

The SSO (with type $\alpha$ ), anticipating the choice of $c^{*}$, therefore ought to endorse the technology if and only if

$$
a+b+c^{*}+\alpha \pi\left(c^{*}\right) \geq 0 .
$$

And so

$$
m\left(b^{*}\right)-b^{*}=\alpha \pi\left(m^{-1}\left(-\left(a+m\left(b^{*}\right)\right)\right)\right) .
$$

This equation is, however, the same, for a given $\alpha$, as the one obtained by combining (4) and (5).

Proposition 8 The outcome is unchanged if the sponsor is unable to commit to the specification $c$.

- Application to licensing fees.

Sponsors sometimes benefit directly (through licensing fees) and not only indirectly (through related activities) from standards. For example, Qualcomm's earnings from technology licensing, which largely derived from transactions involving its patents critical to the CDMA standard, were $\$ 325$ million in the first quarter of fiscal year 2004 and accounted for more than half of the firm's earnings. ${ }^{17}$ Suppose that the sponsor can commit to a licensing fee. ${ }^{18}$ When the sponsor charges licensing fee $p \geq 0$ to users, ${ }^{19}$ then $c=-p$. The users' utility is

$$
a+b-p
$$

\footnotetext{
${ }^{17}$ http: // www.qualcomm.com/press/PDF/QCOM_Q104.pdf (accessed March 28, 2004).

${ }^{18}$ In practice, the commitment made by licensors regarding royalties in standard setting processes is usually vaguer (e.g., RAND). But the sponsor(s) can commit in other ways, for example through a patent pool that sets and commits to a level of royalties.

${ }^{19}$ We assume that negative licensing fees $(p<0)$ cannot be offered as they induce non-serious licensees to accept the cash payment without using the technology.
} 
and the sponsor's utility in case of adoption is

$$
\pi=\pi_{0}+p
$$

And so, $\pi^{\prime}=-1$. Applying equation (5) yields:

Proposition 9 If $\alpha>1$ in the absence of licensing fee, then the optimal licensing fee is positive $(p>0)$, and the sponsor chooses a balanced SSO: $\alpha=1$, yielding socially efficient recommendations $\left(a+b^{*}+\pi=0\right)$. If $\alpha<1$ in the absence of licensing fee, then there is no licensing fee chosen $(p=0)$ even when such fees are feasible.

- Social optimality

As in Section 3, we can investigate the assessment, by a social planner putting relative weight $w$ on the sponsor's interests, of the specification chosen by the sponsor.

Let us consider the following timing:

(1) The SSO (i.e., $\alpha$ ) is chosen by the sponsor (no regulation of SSO choice) or by the social planner (regulation of SSO choice).

(2) The sponsor (in the absence of mandated levels of concessions) or the social planner (under regulation) chooses concession level $c$. The SSO learns $b$ and chooses whether to recommend the standard.

(3) Users decide whether to adopt the standard.

Proposition 10 Consider a social planner putting relative weight $w$ on the sponsor's interests relative to the users'.

(i) There exists a threshold $a_{0}$ such that when the standard is less attractive than $a_{0}$ $\left(a \leq a_{0}\right)$, then laissez-faire is optimal.

(ii) For $a>a_{0}$, regulation of both $S S O$ choice and specification raises social welfare. However, regulation of a single dimension (SSO choice or specification) lowers welfare. 
Proof: Let $\left(\alpha^{*}(a), c^{*}(a)\right)$ denote the unregulated outcome . The social planner by contrast would like to choose $c=\widehat{c}$ with

$$
1+w \pi^{\prime}(\widehat{c})=0
$$

and would like the standard to be adopted whenever

$$
a+b+\widehat{c}+w \pi(\widehat{c}) \geq 0
$$

Let $a_{0}$ be such that $\alpha^{*}\left(a_{0}\right)=w$. For attractiveness $a_{0}$, the social planner does not need to regulate either choice by the sponsor. When $a<a_{0}$, the social planner cannot improve on the sponsor's choices. ${ }^{20}$

So, let us assume that $a>a_{0}$, or equivalently, that $\alpha^{*}(a)>w$. If the social planner regulates the choice of SSO only, at some level $\alpha<\alpha^{*}(a)$, then the sponsor lowers $c$ so as to keep users indifferent between adopting or not:

$$
a+m(-(a+c+\alpha \pi(c)))+c=0 .
$$

The sponsor is worse off than under laissez-faire and the users no better off. Similarly, a regulation of $c$ above $c^{*}(a)$ leads to an increase in $\alpha$ that keeps users indifferent, and to a reduction in social welfare.

\footnotetext{
${ }^{20}$ Using a mechanism design approach, the social planner's optimal scheme solves:

$$
\begin{aligned}
& \text { s.t. } \\
& E[X(b)(a+b+c)] \geq 0 .
\end{aligned}
$$$$
\max _{\{X(\cdot), c\}} E[X(b)[a+b+c+w \pi(c)]]
$$

Letting $\mu$ denote the shadow price of the constraint, it is easy to see that $\mu>0$ if and only if $a<a_{0}$. In this case,

$$
(1+\mu)+w \pi^{\prime}(c)=0
$$

and the standard is adopted if and only if

$$
(1+\mu)(a+b+c)+w \pi(c) \geq 0 .
$$

The solution is the same as for the sponsor's program:

$$
\begin{aligned}
& \max _{\{X(\cdot), c\}} E[X(b) \pi(c)] \\
& \text { s.t. } \\
& E[X(b)(a+b+c)] \geq 0 .
\end{aligned}
$$




\subsection{Technology morphing by the SSO}

Proposition 7 stated an intriguing result: Under free entry into the SSO market, i.e., when the sponsor can choose any desired degree of congruence $\alpha$, there is no dissonance between the selected SSO and the sponsor concerning the standard's specification. Unlike most other results, this particular result hinges on the free entry assumption. By contrast, an SSO with market power can demand concessions beyond what the sponsor would naturally grant.

Suppose that the only operational SSO has type $\alpha$ smaller than what the sponsor would wish (see below), and that the sponsor must rely on this SSO's certification in order to get the standard adopted.

Simplify the model by assuming two possible values of $c: c_{L}$ or $c_{H}$, with $c_{L}<c_{H}$ with $\pi\left(c_{L}\right)>\pi\left(c_{H}\right)>0$. Suppose that, in a non-bargaining situation (the sponsor chooses $c)$, the sponsor obtains adoption following the SSO's endorsement for either specification but prefers low concessions $\left(c_{L}\right)$. Formally, let $\widehat{b}\left(c_{k}\right), k \in\{L, H\}$, with $\widehat{b}\left(c_{H}\right)<\widehat{b}\left(c_{L}\right)$, be defined by

$$
a+\widehat{b}\left(c_{k}\right)+c_{k}+\alpha \pi\left(c_{k}\right)=0
$$

That the sponsor can get adoption regardless of the choice of specification means that

$$
a+m\left(\widehat{b}\left(c_{k}\right)\right)+c_{k} \geq 0 \quad \text { for } \quad k \in\{L, H\}
$$

The sponsor's preference for $c_{L}$ in the static game can be written as:

$$
V^{*}=\left[1-F\left(\widehat{b}\left(c_{L}\right)\right)\right] \pi\left(c_{L}\right)>\left[1-F\left(\widehat{b}\left(c_{H}\right)\right)\right] \pi\left(c_{H}\right)
$$

(If this condition is not satisfied, it can be shown that the sponsor is best off making the high concession at the start of the bargaining game below.) The SSO's preference for high concessions can be expressed as:

$$
c_{H}+\alpha \pi\left(c_{H}\right)>c_{L}+\alpha \pi\left(c_{L}\right) .
$$


Let us now consider the two-period version of this model, with discount factor $\delta$. In each period $t=1,2$ (but only in period 2 if the standard has not been adopted in period 1 , as an adoption decision is irreversible):

- first, the sponsor sets the level of concession $c_{t}$;

- second, the SSO decides whether to recommend the associated standard;

- third, the users decide whether to adopt the standard.

We focus on pure-strategy equilibria of this game.

This bargaining game between sponsor and SSO is reminiscent of the wars of attrition beteween two competing sponsors studied in Farrell (1996) and Simcoe (2003). A novel ingredient is the presence of a third player, the users, who watch the bargaining game and ultimately determine whether the technology is adopted.

Let $\widehat{b}>\widehat{b}\left(c_{L}\right)$ be such that for $b=\widehat{b}$, the SSO is indifferent between endorsing a low-concession standard at date 1 and waiting for a larger concession:

$$
(1+\delta)\left[a+\widehat{b}+c_{L}+\alpha \pi\left(c_{L}\right)\right]=\delta\left[a+\widehat{b}+c_{H}+\alpha \pi\left(c_{H}\right)\right]
$$

And let $\mu\left(b_{1}, b_{2}\right)$ denote the mean of $b$ conditional on $b$ belonging to the interval $\left[b_{1}, b_{2}\right]$.

Suppose that the sponsor chooses $c=c_{L}$ at date 1 . There can be three types of continuation equilibria:

(1) In the first case, the sponsor will not be able to get the standard adopted (even by choosing $\left.c=c_{H}\right)$ at date 2 . In this case, the date- 1 cut-off is $\widehat{b}\left(c_{L}\right)$. The sponsor then gets payoff $(1+\delta) V^{*}$. A necessary and sufficient condition for this intransigent equilibrium to exist is that

$$
a+\mu\left(\widehat{b}\left(c_{H}\right), \widehat{b}\left(c_{L}\right)\right)+c_{H} \leq 0
$$

(The sponsor does not get a second chance, and therefore is able to commit not to make a further concession.)

As $\alpha$ grows, both $\widehat{b}\left(c_{H}\right)$ and $\widehat{b}\left(c_{L}\right)$ decrease and so (6) is more likely to be satisfied. 
For $\alpha=0, a+\widehat{b}\left(c_{H}\right)+c_{H}=0$ and so (6) is not satisfied. For $\alpha \geq \widehat{\alpha}$, where

$$
c_{L}+\widehat{\alpha} \pi\left(c_{L}\right)=c_{H}+\widehat{\alpha} \pi\left(c_{H}\right)
$$

the SSO does not endorse the standard in period 2 if it has not endorsed it in period 1. Hence there exists $\alpha_{1}, 0<\alpha_{1}<\widehat{\alpha}$, such that the intransigent equilibrium exists if and only if $\alpha \geq \alpha_{1}$.

(2) In the second case, the standard will be adopted with some probability at date 2 (which requires making concession $c_{2}=c_{H}$ ). The date 1 cut-off is then $\widehat{b}$, and furthermore:

$$
a+\mu\left(\widehat{b}\left(c_{H}\right), \widehat{b}\right)+c_{H} \geq 0
$$

The discount factor between the two periods $\delta$ must satisfy

$$
(1+\delta)\left[a+m(\widehat{b})+c_{L}\right] \geq \delta\left[a+m(\widehat{b})+c_{H}\right]
$$

otherwise the users would benefit from waiting until date 2 for a larger concession even after the SSO endorses the standard at date 1.

As for the intransigent equilibrium, let us investigate the impact of $\alpha$ on the existence of the technology morphing equilibrium. First, note that $\widehat{b}\left(c_{H}\right)$ and $\widehat{b}$ both decrease with $\alpha$ and so (7) is less likely to be satisfied as $\alpha$ grows. Condition (8) can be rewritten as

$$
m(\widehat{b})-\widehat{b} \geq \alpha\left[(1+\delta) \pi\left(c_{L}\right)-\delta \pi\left(c_{H}\right)\right]
$$

with $\widehat{d b} / d \alpha=-\left[(1+\delta) \pi\left(c_{L}\right)-\delta \pi\left(c_{H}\right)\right]$, and so (9) is also less likely to be satisfied as $\alpha$ grows. Furthermore, both (7) and (9) are satisfied for $\alpha=0$. For $\alpha=\widehat{\alpha}$, the technology morphing equilibrium cannot exist since the SSO can reach its maximum payoff by endorsing the standard at $t=1$ whenever $b \geq \widehat{b}\left(c_{L}\right)$. So there exists $\alpha_{2}$, $0<\alpha_{2}<\widehat{\alpha}$, such that the technology morphing equilibrium exists if and only if $\alpha \leq \alpha_{2}$.

We then obtain a technology morphing equilibrium in which the SSO with positive probability obtains further concessions (with delay) from the sponsor. 
The sponsor's payoff is then

$$
[1-F(\widehat{b})](1+\delta) \pi\left(c_{L}\right)+\left[F(\widehat{b})-F\left(\widehat{b}\left(c_{H}\right)\right)\right] \delta \pi\left(c_{H}\right)
$$

(3) Last, let us investigate the third form of equilibrium, in which the users do not adopt the standard at date 1 regardless of the recommendation. The sponsor must then set $c_{H}$ at date 2 (if no further concession were expected, the standard might as well be adopted at date 1). We use a slight refinement of perfect equilibrium, trembling hand perfect equilibrium, in order to identify the SSO's date-1 behavior. For an arbitrary small probability that the standard is adopted at date 1 in case of endorsement by the SSO, then the SSO endorses the standard if and only if $b \geq \widehat{b}$. Hence this "late adoption equilibrium" exists if and only if (7) is satisfied and (8) violated. For such parameter configurations, the sponsor is better off making concession $c_{H}$ at date 1 already.

To sum up, the intransigent equilibrium exists if (6) is satisfied, the technology morphing equilibrium exists if (7) and (8) are satisfied, and the late adoption continuation equilibrium exists if (7) is satisfied and (8) violated. If (8) is satisfied, and given that (7) is satisfied at $\alpha=\alpha_{1}$, multiple equilibria co-exist: If the SSO expects the users to adopt the high-concession standard at date 2, then the SSO is less prone to endorse the lowconcession standard at date 1 , and so the signal conveyed by a lack of date- 1 endorsement is not as bad, making users willing to adopt the standard at date 2 .

Interestingly, the intransigent equilibrium is less likely to exist, and the technology morphing equilibrium more likely to exist, when the technology becomes more attractive ( $a$ grows). An attractive technology makes it more likely that future concessions trigger adoption despite the users' reluctance to adopt given the lack of endorsement at date 1. This in turn undermines the credibility of the sponsor sticking to a user-unfriendly specification.

We can also illustrate equilibrium behavior as a function of $\alpha$. For this, assume that discounting is sufficient, so that (8) holds (this rules out the late-adoption continuation equilibrium, but the latter is uninteresting to the extent that the sponsor is better off 
making the concession at date 1 already). Using (6) and (7), which do not depend on the discount factor, then the behavior following a low concession $c_{L}$ at date 1 is depicted as in figure 1. It can further be shown that either of the two behaviors depicted in figure 1 can be observed once the sponsor's choice of date 1 concession is endogenized. ${ }^{21}$ intransigent equilibrium

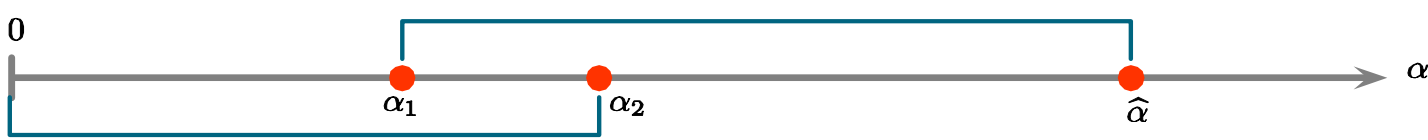

technology morphing equilibrium

\section{Figure 1}

Proposition 11 When the SSO is more user-oriented than the sponsor would wish, the SSO engages with the sponsor in a two-way bargaining game over standard design, with a third party, the users, observing the game and ultimately determining adoption. In an intransigent equilibrium, the sponsor can credibly commit to a low-concession level, thanks to the "rejection curse," which discourages users from later accepting even a higher concession when the SSO fails to endorse the initial design. By contrast, in a technology-morphing equilibrium, the SSO may force concessions on the sponsor. There exists $\left(\alpha_{1}, \alpha_{2}\right)$ in $(0, \widehat{\alpha})$ such that the intransigent equilibrium exists if and only if $\alpha \geq \alpha_{1}$, and the technology morphing equilibrium exists if and only if $\alpha \leq \alpha_{2}$. The two equilibria co-exist over a range of parameters.

\section{Extensions}

This section investigates a number of relevant extensions.

\footnotetext{
${ }^{21}$ Suppose, first, that $\alpha=\widehat{\alpha}$. Then $\widehat{b}\left(c_{L}\right)=\widehat{b}\left(c_{H}\right)$, and so the sponsor's payoff, $\left[1-F\left(\widehat{b}\left(c_{L}\right)\right)\right] \pi\left(c_{L}\right)$, when choosing $c_{L}$ exceeds her payoff, $\left[1-F\left(\widehat{b}\left(c_{H}\right)\right)\right] \pi\left(c_{H}\right)$, when choosing $c_{H}$. So, for $\alpha$ equal or close to $\widehat{\alpha}$, users adopt technology $c_{L}$ or never adopt.

Next, assume that $\alpha=0$. For small $\delta$, then $\widehat{b}$ is close to $\widehat{b}\left(c_{L}\right)$ and so the profit in the technology morphing equilibrium exceeds that associated with the choice of $c_{H}$ at date 1.
} 


\subsection{Downstream presence of the sponsor}

Assume now that the sponsor is herself a user and in fact represents a fraction $\theta \in[0,1]$ of the user community. In a first step, let us rule out royalties and assume that the sponsor's payoff in case of adoption is

$$
\pi+\theta(a+b)
$$

while the independent users' payoff is

$$
(1-\theta)(a+b)
$$

For simplicity, we assume that these payoffs are conditional on all users adopting (strong network externalities). The upstream profit $\pi$ represents the spillover of the downstream adoption on a complementary activity of the sponsor.

As earlier, let $X(b) \in[0,1]$ denote the probability of adoption by the user community. An upper bound on the sponsor's profit is

$$
\begin{aligned}
& \max _{\{X(\cdot)\}} E[X(b)[\pi+\theta(a+b)]] \\
& \text { s.t. } \\
& E[X(b)[a+b]] \geq 0 .
\end{aligned}
$$

This is equivalent to selecting a threshold above which the users adopt the technology. As earlier, let $b^{*}$ be defined by:

$$
a+m\left(b^{*}\right)=0
$$

For an arbitrary cut-off $\widehat{b}$, the sponsor's profit is:

$$
[1-F(\widehat{b})][\pi+\theta[a+m(\widehat{b})]]
$$

Ignoring the users' participation constraint, taking the log-derivative and using the expression for $m^{\prime}$ shows that this profit is maximized for cut-off $b^{* *}$ given by:

$$
\pi+\theta\left(a+b^{* *}\right)=0
$$


as one would expect.

Reintroducing the users' participation constraint, we are led to consider two cases: Weak downstream presence: $\pi+\theta\left[a+m^{-1}(-a)\right]>0$. Recalling that $a+m^{-1}(-a)<0$, this condition is satisfied for $\theta$ below some strictly positive threshold. When the sponsor has a weak downstream presence, then $b^{* *}<b^{*}$, and so the sponsor's preferred policy is too lax to enlist the independent users. Thus, the "constrained optimal" policy is given by threshold $b^{*}$.

This policy can be implementing by selecting an SSO putting relative weight $\alpha$ on the sponsor relative to independent users, where

$$
(1-\theta)\left(a+b^{*}\right)+\alpha\left[\pi+\theta\left(a+b^{*}\right)\right]=0,
$$

or

$$
a+b^{*}+\gamma \pi=0
$$

where

$$
\gamma \equiv \frac{\alpha}{1-\theta+\theta \alpha}
$$

$\gamma<1 / \theta$ as long as $\theta<1$. Using the first equality, it can be checked that $\alpha$ grows from $\gamma>1$ to $\infty$ as $\theta$ grows in this region if $\alpha>1$ in the absence of downstream presence $(\theta=0)$ and decreases as $\theta$ grows if $\alpha<1$ in the absence of downstream presence.

Strong downstream presence: $\pi+\theta\left[a+m^{-1}(-a)\right]<0$. This region exists only if $\pi$ is not too large (i.e., $\pi<-a-m^{-1}(-a)$ ). Put another way, it exists if and only if $\alpha>1$ in the absence of downstream presence $(\theta=0)$. In this region, $b^{* *}>b^{*}$, and so the independent users' participation constraint is not binding. The sponsor is sufficiently preoccupied with downstream performance that independent users obtain a rent. This does not mean, though, that the sponsor and the independent users are fully congruent; indeed, the sponsor selects an SSO that issues recommendations that are still too favorable to the sponsor's interests: Namely, the selected SSO recommends adoption whenever $b \geq b^{* *}$ where $a+b^{* *}<0$. The selected SSO is entirely devoted to the sponsor's interests $(\alpha=\infty)$. 
In a sense, the SSO is basically a sponsor-controlled laboratory that advises the sponsor (and indirectly the independent users) as to the adequacy of the technology.

We summarize these results in:

Proposition 12 Suppose that the sponsor represents a fraction $\theta \in[0,1]$ of the downstream industry and that there are strong network externalities.

- If $\pi+\left[a+m^{-1}(-a)\right]<0$, that is if $\alpha>1$ in the absence of downstream presence, there exists $\theta^{*}<1$ such that:

(i) for weak downstream presence $\left(\theta \leq \theta^{*}\right)$, the independent users' participation constraint is binding. The selected SSO recommends adoption if and only if $b \geq b^{*}$, and puts more and more weight on the sponsor's interests as the latter's downstream presence increases.

(ii) for strong downstream presence $\left(\theta>\theta^{*}\right)$, independent users enjoy a rent. The selected SSO is entirely devoted to the sponsor's interests.

- If $\pi+\left[a+m^{-1}(-a)\right]>0$, i.e., if $\alpha$ is smaller than 1 in the absence of downstream presence, then the independent users' participation constraint is always binding, and the selected SSO puts less and less weight on the sponsor's interests as the latter's downstream presence increases.

For completeness, let us describe the outcome when, as in Section 4, the sponsor derives income from royalties: $\pi=\pi_{0}+p$, where $\pi_{0}$ is the non-royalty-based spillover profit from adoption. Then the sponsor's total payoff for a threshold $\widehat{b}$ is

$$
[1-F(\widehat{b})]\left[\pi_{0}+\theta[a+m(\widehat{b})]+(1-\theta) p\right]
$$

where $p=a+m(\widehat{b})$. It is easy to check that, as in Proposition 9, the sponsor wants to generate cut-off rule $a+\widehat{b}+\pi_{0}=0$.

Proposition 4 showed that in the absence of downstream presence, allowing the sponsor to receive a private signal about user benefit has no impact on equilibrium. This is no 
longer the case under downstream presence, at least if $\theta$ is sufficiently large. ${ }^{22}$ Intuitively, when confident that user benefits are high, it is relatively costless for the sponsor to turn to an SSO that is inclined to accept standards that are weak, even from the sponsor's viewpoint.

To demonstrate this, let us assume that the sponsor's signal makes her optimistic or pessimistic about user benefits: With probability $\rho$ (respectively, $1-\rho$ ), the density of her distribution conditional on the signal is $f_{H}(b)$ (respectively $f_{L}(b)$ ). We assume the monotone likelihood ratio property:

$$
\text { (MLRP) } \quad \frac{f_{H}(b)}{f_{L}(b)} \text { is strictly increasing in } b .
$$

MLRP implies that under symmetric information (users too receive the sponsor's signal), the optimistic sponsor would select a more complacent SSO under weak downstream presence:

$$
b_{H}^{*}<b_{L}^{*}
$$

(where $b_{i}^{*}$ is defined by $a+m_{i}\left(b_{i}^{*}\right)=0$, and $m_{i}$ is relative to the distribution $f_{i}, i=H, L$ ).

Under asymmetric information, we look for a separating equilibrium, assuming that the pessimistic type has weak downstream presence $\left(b_{L}^{*}>b^{* *}\right) .{ }^{23}$ In a separating equilibrium, the choice of SSO reveals the sponsor's type. It is therefore optimal for the pessimistic type to choose the same SSO that she would choose under symmetric information. And so the cutoff is $b_{L}^{*}$.

Let $b_{H}$ denote the cut-off of the SSO chosen by the optimistic type. The following incentive compatibility condition needs to be satisfied in order for the equilibrium to be separating:

$$
\int_{b_{L}^{*}}^{\infty}[\pi+\theta(a+b)] f_{L}(b) d b \geq \int_{b_{H}}^{\infty}[\pi+\theta(a+b)] f_{L}(b) d b .
$$

\footnotetext{
${ }^{22}$ We are grateful to Emmanuel Fahri for Proposition 13 and its proof.

${ }^{23}$ Because $m_{H}^{-1}(y)<m_{L}^{-1}(y)$ for all $y$, this condition says nothing about whether the optimistic type has a strong or weak downstream presence.
} 
Because $[\pi+\theta(a+b)]$ is positive above $b^{* *}$, incentive compatibility requires that:

$$
b_{H}<b^{* *} \text {. }
$$

This condition in turn requires that the optimistic type have a strong downstream presence $\left(b_{H}^{*}<b^{* *}\right)$; otherwise, independent users would not adopt the standard even when they learn that the sponsor is optimistic and the SSO recommends the standard. Thus, $\theta$ must satisfy:

$$
\pi+\theta\left[a+m_{H}^{-1}(a)\right]<0 .
$$

In the "least cost separation equilibrium" (i.e., the separating equilibrium that is Pareto-dominant in the class of separating equilibria), (10) is satisfied with equality. ${ }^{24}$ For this separating equilibrium to exist, a necessary and sufficient condition is then that the independent users adopt the standard when they learn that the sponsor is optimistic:

$$
b_{H} \geq b_{H}^{*}
$$

It can be checked that (10) and (11) are not inconsistent.

Proposition 13 Assume that under symmetric information the optimistic type has a strong downstream presence and the pessimistic type a weak downstream presence $\left(b_{H}^{*}<\right.$ $\left.b^{* *}<b_{L}^{*}\right)$. Let $b_{H}<b^{* *}$ be defined by (10) satisfied with equality. Then if $b_{H} \geq b_{H}^{*}$, there exists a separating equilibrium. The sponsor signals that she has good news about users' benefit by choosing a sponsor who puts even more weight on profitability than she herself does; she thereby demonstrates that low user benefits are unlikely.

${ }^{24}$ MLRP then implies that the other incentive constraint is satisfied:

$$
\int_{b_{H}}^{b_{L}^{*}}[\pi+\theta(a+b)] f_{H}(b) d b=\int_{b_{H}}^{b_{L}^{*}}[\pi+\theta(a+b)] \frac{f_{H}(b)}{f_{L}(b)} f_{L}(b) d b \geq 0 .
$$

The separating equilibrium is for example sustained by the belief that the sponsor is pessimistic whenever she selects an SSO with cutoff exceeding $b_{H}$. 
As is usual, the separating equilibrium may be dominated (even for the optimistic type) by a pooling (or semi-separating) equilibrium, which exists if the prior probability $\rho$ that the sponsor is optimistic is large enough. For example, for large enough $\rho$, threshold $b^{* *}$ can be obtained in a pooling equilibrium.

\subsection{User heterogeneity}

Until now we have assumed that any given user group is homogeneous and therefore has all its members adopt or not adopt. Let us now treat the case of within-user-group heterogeneity and allow for network externalities as well. We first analyze user heterogeneity in the absence of network externalities among users (case a), then under "strong network externalities," i.e., when all users must adopt for the standard to be successful (case b), and finally the general case (case c). Note that user homogeneity made the issue of network externalities moot, since all users either adopted or did not adopt.

a) No network externalities.

Assume that there are two types of users. ${ }^{25}$ Type $H$, "eager users", have utility $U_{H}(b)=a_{H}+b$, and are in proportion $\rho$. Type $L$, "skeptical users," have utility $U_{L}(b)=$ $a_{L}+b$, where $a_{L}<a_{H}$, and are in proportion $1-\rho .{ }^{26}$ Users know their type, but the sponsor cannot tell them apart. The sponsor receives $\pi_{H}$ when the eager types adopt and $\pi_{L}$ when the skeptical types adopt (where the proportions $\rho$ and $1-\rho$ are factored into the definitions of $\pi_{H}$ and $\pi_{L}$ ). Adoption by both types yields $\pi=\pi_{H}+\pi_{L}$.

Let us take a mechanism design approach in order to determine an upper bound on the sponsor's profit and then show how this upper bound can be obtained through adequate forum shopping. Let $X_{i}(b) \in[0,1]$ denote the probability that type $i, i \in\{L, H\}$, adopts

\footnotetext{
${ }^{25}$ We are grateful to Emmanuel Fahri for Proposition 14 and its proof.

${ }^{26} \mathrm{We}$ assume that $a_{H}+E(b)<0$, and so no category of users is willing to adopt the standard without certification.
} 
when user benefit is $a_{i}+b$. The sponsor's maximal profit is given by

$$
\begin{aligned}
& \max _{\left\{X_{L}(\cdot), X_{H}(\cdot)\right\}}\left\{E\left[X_{L}(b) \pi_{L}+X_{H}(b) \pi_{H}\right]\right\} \\
& \text { s.t. } \\
& E\left[X_{L}(b)\left(a_{L}+b\right)\right] \geq 0, \\
& \text { and } \\
& E\left[X_{H}(b)\left(a_{H}+b\right)\right] \geq E\left[X_{L}(b)\left(a_{H}+b\right)\right] .
\end{aligned}
$$

The first constraint is the skeptical users' participation constraint (were they to expect a negative payoff, they could improve their welfare by never participating), and the second the eager users' incentive constraint (they can always masquerade as skeptical users). A straightforward analysis of this linear program demonstrates the existence of thresholds $b_{L}$ and $b_{H}$ :

$$
\begin{aligned}
& X_{L}(b)=1 \quad \text { iff } \quad b \geq b_{L} \\
& \text { and } \\
& X_{H}(b)=1 \quad \text { iff } b \geq b_{H} ;
\end{aligned}
$$

furthermore, the two constraints are binding. And so,

$$
b_{L}=b_{L}^{*}
$$

where

$$
a_{L}+m\left(b_{L}^{*}\right)=0 .
$$

The incentive constraint is:

$$
\begin{array}{ccc}
{\left[1-F\left(b_{H}\right)\right]\left[a_{H}+m\left(b_{H}\right)\right]=\left[1-F\left(b_{L}^{*}\right)\right]\left[a_{H}+m\left(b_{L}^{*}\right)\right]} \\
\Longleftrightarrow & {\left[F\left(b_{L}^{*}\right)-F\left(b_{H}\right)\right] a_{H}+\int_{b_{H}}^{b_{L}^{*}} b d F(b)=0} \\
& a_{H}+\mu\left(b_{H}, b_{L}^{*}\right)=0,
\end{array}
$$

where as earlier, $\mu(x, y)$ denote the expectation of $b$ conditional on $b$ belonging to the interval $[x, y]$. 
Note that the existence of a value of $b_{H}$ satisfying (13) requires that

$$
a_{H}+b_{L}^{*} \geq 0
$$

Conversely, if $a_{H}+b_{L}^{*} \geq 0$, then there exists a $b_{H}$ satisfying (13) (as long as the standard cannot be adopted as a de facto standard, $\left.a_{H}+\mu\left(-\infty, b_{L}^{*}\right)<0\right)$.

When $a_{H}+b_{L}^{*}<0$, the incentive compatibility condition (13) requires that $b_{H}>b_{L}^{*}$, which is infeasible as long as all users receive the same information: Because eager users by definition are more eager to adopt than skeptical ones, $X_{H}(b) \geq X_{L}(b)$ for all $b$, and so $b_{H} \leq b_{L}^{*}$ after all. The constrained optimum is then $b_{H}=b_{L}^{*}$ as long as $\pi_{L} / \pi_{H}$ is not too small (if $1-\rho$ and therefore $\pi_{L} / \pi_{H}$ are small, it becomes optimal for the sponsor to ignore the skeptical users and to choose $b_{H}=b_{H}^{*}$, with $\left.a_{H}+m\left(b_{H}^{*}\right)=0\right)$.

Let us now turn to the implementation of the optimal policy. Let us first dispose of the uninteresting latter case in which $b_{H}=b_{L}^{*}$. The sponsor then should select an SSO with cutoff $b_{L}^{*}$, i.e., she should focus on skeptical users and ignore eager ones. ${ }^{27}$ Similarly, if the sponsor does not try to appeal to skeptical types, and so $b_{H}=b_{H}^{*}$, the SSO optimally selects an SSO with cutoff $b_{H}^{*}$.

More interestingly, suppose that $a_{H}+b_{L}^{*}>0$ and so

$$
b_{H}<b_{L}^{*}
$$

One implementation consists in applying to two SSOs. SSO $i(i \in\{L, H\})$ involves users of type $i$ and the sponsor and has objective function $U_{i}+\alpha_{i} \pi$, where

$$
a+b_{i}+\alpha_{i} \pi_{i}=0
$$

It is then an equilibrium for SSOs to recommend adoption when $b \geq b_{i}$. When $b<b_{L}$, SSO $H$ knows that SSO $L$ will not recommend adoption. Hence, SSO $H$ recommends

\footnotetext{
${ }^{27}$ If the SSO does not recommend adoption, even eager users will not adopt the standard as

$$
a_{H}+\mu\left(-\infty, b_{L}^{*}\right)<a_{H}+b_{L}^{*}<0 .
$$
}


adoption if and only if

$$
a_{H}+b+\alpha_{H} \pi_{H} \geq 0 \Longleftrightarrow b \geq b_{H}
$$

Similarly, when $b>b_{H}$, SSO $L$ knows that SSO $H$ will recommend adoption. Hence, SSO $L$ recommends adoption if and only if

$$
a_{L}+b+\alpha_{L}\left(\pi_{L}+\pi_{H}\right) \geq \alpha_{L} \pi_{H} \Longleftrightarrow b \geq b_{L}=b_{L}^{*}
$$

The implementation through two user-specific SSOs is by no means unique. Indeed, the optimum can also be implemented through a single SSO, with preferences

$$
\pi+\beta_{L} U_{L}+\beta_{H} U_{H}
$$

where $\pi_{L}+\beta_{L}\left(a_{L}+b_{L}^{*}\right)=0$ and $\pi_{H}+\beta_{H}\left(a_{H}+b_{H}\right)=0$.

Proposition 14 Suppose that users decompose into eager users (with utility $a_{H}+b$ ) and skeptical ones (with utility $a_{L}+b$ ), and that user type is private information. In the interesting case, in which the sponsor tries to attract both types of users, then

$$
b_{L}=b_{L}^{*} \text { where } a_{L}+m\left(b_{L}^{*}\right)=0 .
$$

Furthermore, and provided that $a_{H}+b_{L}^{*}>0$, then the eager types adopt if and only if $b \geq b_{H}$ with $b_{H}<b_{L}^{*}$ is given by

$$
a_{H}+\mu\left(b_{H}, b_{L}^{*}\right)=0 \text {. }
$$

The optimal policy can be implemented either by using two user-specific SSOs or else a single SSO defending both categories of users.

b) Strong network externalities: standards as multi-sided markets

Standards may be adopted by multiple groups of users. Indeed, sponsors often aim at "getting multiple sides on board." ${ }^{28}$ For example, one key challenge faced by the MPEG2

\footnotetext{
${ }^{28}$ To use a standard expression from the multi-sided markets literature (e.g., Rochet-Tirole (2003)).
} 
standard was to attract both the consumer electronics and the computer manufacturers groups, while providing sponsors with sufficient royalty income. The patent-holders found the consumer electronics industry to be more comfortable with paying royalties for patented material in standards and more focused on assuring interoperability and a critical mass of users. The computer industry was much more reluctant to license the patents, having set standards on its own for many years and being accustomed to royalty-free cross-licensing agreements between industry members.

Suppose that there are $n$ groups of users, $i=1, \cdots, n$, and that the standard is successful (in which case the sponsor receives $\pi$ ) if and only if each of these $n$ groups adopts the standard (strong network externalities are present). In other words, the standard is really adopted only if all user groups are taken on board. Leaving aside for the moment licensing fees and other standard specifications, group $i$ 's utility from adopting the standard (when the $n-1$ other groups adopt it as well) is $a_{i}+b_{i}$, where, as earlier, $a_{i}$ is known and $b_{i}$ is not. The state of nature $b=\left(b_{1}, \cdots, b_{n}\right)$ is distributed according to some joint distribution $F(b)$. We assume that group $i$ 's utility when it adopts and less than $(n-1)$ other groups adopt as well is always negative, so that it is not feasible to have less than the full set of users adopt.

We first ask whether the sponsor should go to $n$ different SSOs, for example each one trusted by one specific user group, or to a single SSO. The need for coordination calls for the use of a single SSO:

Proposition 15 It is optimal for the sponsor to apply to a single SSO.

Proof: Let $b=\left(b_{1}, \cdots, b_{n}\right)$ and let $X(b)$ denote the (present discounted) probability of adoption by the (full set of ) users. An upper bound on the sponsor's payoff is given by:

$$
\begin{aligned}
& \max _{\{X(\cdot)\}} E[X(b) \pi] \\
& \text { s.t. } \\
& E\left[X(b)\left(a_{i}+b_{i}\right)\right] \geq 0 \text { for } i=1, \cdots, n .
\end{aligned}
$$


Letting $\beta_{i}$ denote the shadow prices of the constraints, the standard should be adopted if and only if

$$
\pi+\sum_{i=1}^{n} \beta_{i}\left(a_{i}+b_{i}\right) \geq 0
$$

The sponsor can reach this upper bound by applying to an SSO with relative weights $\left(1, \beta_{1}, \cdots, \beta_{n}\right)$ on the sponsor and the users' groups.

Applying to multiple SSOs (with "non-colinear" preferences) by contrast does not yield this "interdependent" decision rule: For example, an SSO that would put relative weights 1 on the sponsor, $\beta_{i}$ on user group $i$, and 0 on the other user groups would recommend adoption to group $i$ if and only if $\pi+\beta_{i}\left(a_{i}+b_{i}\right) \geq 0$, regardless of the values of $b_{j}, j \neq i$.

Second, we generalize Proposition 9 to multiple groups of users (under strong network externalities): If the sponsor can perfectly capture users' surpluses through licensing fees, then it is optimal for the sponsor to choose a balanced SSO. Furthermore, we provide sufficient conditions under which the user groups who find the technology least attractive are charged less:

Proposition 16 Suppose that groups of users have different $a_{i} s$, that the $b_{i} s$ are independently and identically distributed, and that the sponsor can discriminate in her choice of licensing fees $\left(p_{1}, \cdots, p_{n}\right)$ to users. Then, provided that $p_{i}>0$ for all $i$

(i) the sponsor selects a balanced SSO (putting equal weight on all parties),

(ii) if the distribution $F(b)$ is symmetrical, then the sponsor sets lower licensing fees to groups that are more reluctant to adopt the standard; indeed, $a_{i}-p_{i}$ is the same for all groups.

Proof: The proof of part (i) of Proposition 16 is a simple extension of that of Proposition 9. Part (ii) results from the fact that $p_{i}=a_{i}+E\left(b_{i} \mid\right.$ endorsement). By symmetry of the distribution and given that $\beta_{i}=1$ for all $i$, the expectation of $b_{i}$ conditional on SSO 
endorsement is the same for all groups of users.

c) The general case.

Consider an heterogeneous group with a large number (indeed a continuum) of members indexed by $i .^{29}$ Member $i$ 's net utility from adopting the standard is

$$
U_{i}(x)=a(x)+b-\varepsilon_{i},
$$

where $x \in[0,1]$ is the fraction of members adopting the standard, and the idiosyncratic preference shock $\varepsilon_{i} \in(-\infty,+\infty)$ is distributed according to some cumulative distribution $G(\cdot)$, with density $g(\cdot)$. Positive network externalities correspond to $a^{\prime}(x)>0$. In order to abstract from technical complications arising from multiple equilibria, we will assume that network externalities are not too strong: $1>g\left(G^{-1}(x)\right) a^{\prime}(x)$ for all $x$. The sponsor's profit is denoted $\pi(x)$ and is assumed to increase with $x$.

Consider the choice made by a SSO putting relative weight $\alpha$ on the sponsor's interests. Suppose that it endorses the standard whenever $b \geq b^{*}$. Then user $i$ adopts the standard following the endorsement if and only if

$$
a(x)+m\left(b^{*}\right)-\varepsilon_{i} \geq 0
$$

and thus the fraction $x$ of adopters is given by

$$
x=G\left(a(x)+m\left(b^{*}\right)\right),
$$

which has a unique solution $x^{*}$ (increasing in $\left.m\left(b^{*}\right)\right)$ from our assumption). This yields the equilibrium adoption rate:

$$
x^{*}=G\left(a\left(x^{*}\right)+m\left(b^{*}\right)\right)
$$

The SSO recommends adoption if and only if

$$
\begin{aligned}
\int_{-\infty}^{G^{-1}\left(x^{*}\right)} & {\left[a\left(x^{*}\right)+b-\varepsilon\right] d G(\varepsilon)+\alpha \pi\left(x^{*}\right) } \\
= & {\left[a\left(x^{*}\right)+b-\mathcal{E}\left(x^{*}\right)\right] x^{*}+\alpha \pi\left(x^{*}\right) \geq 0 }
\end{aligned}
$$

\footnotetext{
${ }^{29}$ The case of absence of network externalities (case a) corresponds to $a(x)$ independent of $x$; that of strong network externalities (case b) to $a(x)=-\infty$ unless $x=1$ (one must then take a bounded support for $\varepsilon)$.
} 
where $\mathcal{E}\left(x^{*}\right)=\left[\int_{-\infty}^{G^{-1}\left(x^{*}\right)} \varepsilon d G(\varepsilon)\right] / x^{*}$ is the average idiosyncratic shock of adoptors; this condition is equivalent to:

$$
b \geq \mathcal{E}\left(x^{*}\right)-a\left(x^{*}\right)-\frac{\alpha \pi\left(x^{*}\right)}{x^{*}} \equiv b^{*} .
$$

Together, (14) and (15) define $b^{*}$ and $x^{*}$ for a given $\alpha$.

Proposition 17 In the presence of user heterogeneity and network externalities,

(i) the equilibrium, for a given $\alpha$, is given by

$$
x^{*}=G\left(a\left(x^{*}\right)+m\left(b^{*}\right)\right)
$$

and

$$
b^{*}=\mathcal{E}\left(x^{*}\right)-a\left(x^{*}\right)-\frac{\alpha \pi\left(x^{*}\right)}{x^{*}}
$$

(ii) an increase in the intensity of network externalities (i.e., an increase in $a^{\prime}\left(x^{*}\right)$ keeping $a\left(x^{*}\right)$ constant) calls for an application to a tougher SSO (a lower $\alpha$ ).

Proof: (i) has already been proved. To prove (ii), note that the sponsor solves

$$
\max \left\{\left[1-F\left(b^{*}\right)\right] \pi\left(x^{*}\right)\right\}
$$

where $b^{*}$ and $x^{*}$ are given in (i). The result is obtained through differentiation.

\section{Competing standards and strategic forum shop- ping}

As we discussed, competition weakens the case for a given standard. If the rival technology provides users with a known and positive utility, contemporaneous rivalry can be modeled as a decrease in the strength $a$ of the standard; potential future contestability can be similarly analyzed as a reduction in $a$.

We now consider strategic forum shopping by two rival technologies $(j=1,2)$. These technologies, if adopted, deliver $a_{j}+b_{j}$ to users and $\pi$ to the sponsor, where as earlier 
$a_{j}$ is known and $b_{j}$ is not. Such rivalry raises a host of new and complex issues: Is it still optimal for a sponsor to apply to a single SSO? Should an SSO still render a simple $(0 / 1)$ recommendation or should its opinion be expressed in a more sophisticated manner? Should an SSO render a "myopic" opinion (that is, the recommendation it would issue were the standard the unique possible one)? Will sponsors apply to separate SSOs or to the same one? How are strategies affected by the similarity (correlation) of the two technologies?

Let us analyze this rivalry in a simple symmetric example:

- $\quad b_{j}$ can take one of three values: $-\infty$ (utterly unacceptable), $\underline{b}$, and $\bar{b}>\underline{b}$. Let $x=\operatorname{Pr}$ $(b=\bar{b})$ and $X=\operatorname{Pr}(b=\underline{b}$ or $\bar{b})$;

- users obtain utility $a+b_{j}$ when adopting standard $j$;

- in a first step, we assume that the users would not want to adopt a standard with type $\underline{b}: a+\underline{b}<0$; however,

$$
a+m(\underline{b})=a+\frac{(X-x) \underline{b}+x \bar{b}}{X}>0
$$

- if users perceive two technologies to have equal (and positive) merit, they choose between the two randomly with equal probabilities.

We will say that an SSO is friendly if, on a stand alone basis, it accepts standard $\underline{b}$ (i.e., $a+\underline{b}+\alpha \pi \geq 0)$ and tough if it accepts only standard $\bar{b}(a+\bar{b}+\alpha \pi>0>a+\underline{b}+\alpha \pi)$.

It is never optimal in this example for a sponsor to apply to both a tough and a friendly SSO, if there is at least an infinitesimal cost of application: it obtains the same certification as when it applies only to a tough SSO if it gets accredited by both; on the other hand, the condition $a+\underline{b}<0$ implies that the technology is never adopted if it is not accepted by the tough SSO.

Monopoly standard.

The monopoly benchmark has the sponsor apply to a friendly SSO as

$$
X \pi>x \pi \text {. }
$$


Two rival standards.

There are three potential pure-strategy equilibria of the forum-shopping game between the standard duopolists:

a) Both apply to a tough SSO.

When both search for the highest certification, each gets:

$$
x\left[x \frac{\pi}{2}+(1-x) \pi\right]
$$

A sponsor who would take the less ambitious strategy of applying to a friendly SSO would get:

$$
X(1-x) \pi
$$

Thus, this T-submission equilibrium exists if and only if:

$$
x\left(1-\frac{x}{2}\right) \geq X(1-x) .
$$

b) Both apply to a friendly SSO.

Each sponsor then obtains:

$$
X\left[X \frac{\pi}{2}+(1-X) \pi\right]
$$

while she would obtain $x \pi$ by deviating and applying to a tough SSO. This F-submission equilibrium thus requires that:

$$
X\left(1-\frac{X}{2}\right) \geq x
$$

c) Differentiated strategies.

The sponsor applying to the tough SSO obtains $x \pi$. From the previous analysis, she does not want to apply to a friendly SSO instead as long as

$$
x \geq X\left(1-\frac{X}{2}\right)
$$


The sponsor applying to the friendly SSO obtains $X(1-x) \pi$, which must exceed the payoff, $x\left(x \frac{\pi}{2}+(1-x) \pi\right)$, that she would obtain by deviating and applying to a tough SSO. Hence, this differentiated submissions equilibrium equilibrium also requires that: ${ }^{30}$

$$
X(1-x) \geq x\left(1-\frac{x}{2}\right) .
$$

It can be shown that in this equilibrium the sponsor applying to a tough SSO is better off than his less ambitious rival. We thus obtain three regions. Fixing the probability $X$, and increasing $x$ toward $X,{ }^{31}$ first both apply to friendly SSOs, then the sponsors follow differentiated strategies, and finally both apply to tough SSOs.

Let us next assume that $a+\underline{b}>0$. When $a+\underline{b}>0$, then sponsors may apply to both a tough and a friendly SSO in equilibrium. Because we assumed away application costs (or else posited small ones), applying to both dominates applying only to a tough SSO; for the sponsor thereby gets a "second chance" if her technology is rejected by the tough SSO.

By contrast, applying to both types of SSO does not necessarily dominate applying solely to a friendly SSO because of the by-now familiar stigma attached to being rejected by a tough SSO. Indeed, it is easily seen that the equilibrium is unique and:

- both go for a double submission (to both a tough and a friendly SSO) if and only if

$$
x \geq \frac{X}{2}
$$

(dual-submission equilibrium),

- both apply solely to a friendly SSO (F-submission equilibrium) if and only if

$$
x<\frac{X}{2} .
$$

Figure 2 summarizes the analysis. ${ }^{32}$

\footnotetext{
${ }^{30}$ There exists also a symmetric mixed strategy equilibrium besides the two pure-strategy ones.

${ }^{31} x$ cannot start quite at 0 from the condition $a+m(\underline{b})>0$.

${ }^{32} x_{1}, x_{2}$ and $x_{3}$ are given by:

$$
a+\frac{\left(X-x_{1}\right) \underline{b}+x_{1} \bar{b}}{X}=0,
$$
}




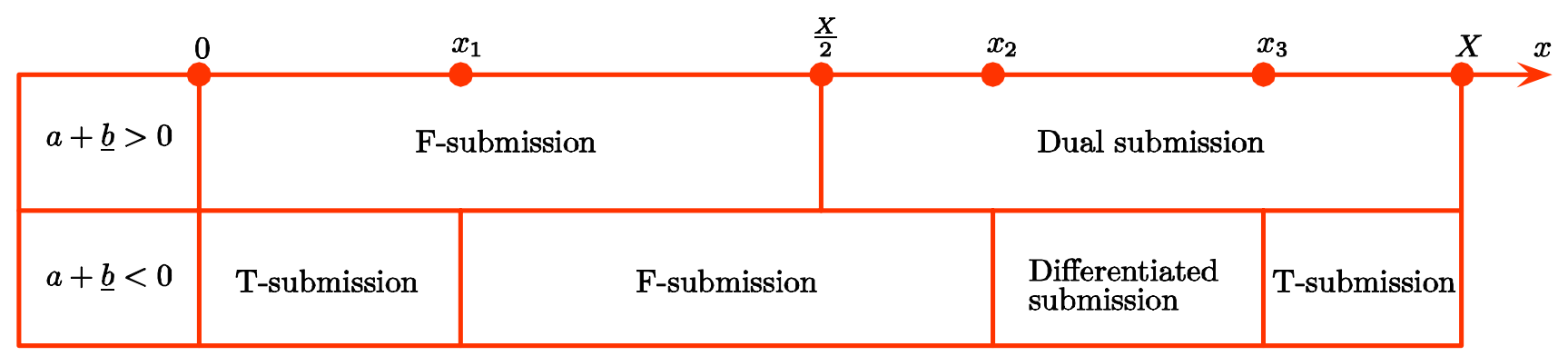

Figure 2

Numerous examples exist where sponsors of competing standards have chosen similar or different strategies when selecting SSOs. As an example of a differentiated strategy, manufacturers of asynchronous transfer mode (ATM) equipment chose to develop their standard for ATM over multi-protocol label switching based networks within their ATM Forum, while the IETF was pursuing a similar project simultaneously. Some observers suggested that the ATM Forum's approach was to essentially clone the functionality of ATM when it ran on dedicated ATM switches to other settings. While this was convenient for the manufacturers, the decision led to added complexity and cost for the network operators. As an example of sponsors choosing very similar strategies, multiple sponsors of wireless Internet connectivity standards applied to the Institute of Electrical and Electronic Engineers (IEEE), where their proposals were considered by different committees within the 802.11 group.

A few comments are in order. First, when $a+\underline{b}>0$, the game between the two sponsors

$$
x_{2}=X\left(1-\frac{X}{2}\right)
$$

and

$$
x_{3}\left(1-\frac{x_{3}}{2}\right)=X\left(1-x_{3}\right)
$$

Note that $x_{1}$ depends on $a$, and that for $a+\underline{b}$ negative, but converging to 0 , the left-handside T-submission region vanishes.

When $a+\underline{b}<0$ and $x<x_{1}$, the equilibrium is a T-submission one since $a+m(\underline{b})<0$ in this region. 
is a zero-sum game (from their point of view). The probability that one of the standards is adopted is $1-(1-X)^{2}$ and is independent of their forum shopping strategies. Users, though, are not indifferent. They are always better off when they take better informed decisions, i.e., when sponsors engage in dual submissions. The equilibrium payoffs are then unique, a well-known property of zero-sum games.

By contrast, when $a+\underline{b}<0$, the game between the sponsors no longer is a zerosum game. Going for an endorsement by a tough SSO reduces the overall probability of adoption of a standard, and therefore must come at the expense of the rival sponsors' welfare. This negative externality on the rival sponsor gives rise to "holier-than-thou" strategies, or a form of "prisoner's dilemma". Again, this is all seen from the sponsors' perspective. Users prefer sponsors to apply to tough SSOs.

Last, comparing the behavior of a sponsor when she faces no competition and when she does, note that standards competition always leads to applications to weakly less friendly SSOs. The "weakly" in the previous sentence is actually an artifact of the discreteness of this version of the model. Indeed, if as in the rest of the paper, we had assumed that $b$ is continuous, it is easy to see that both sponsors' applying to SSOs with type $\alpha^{*}(a)$ (giving rise to cut-off $b^{*}$ such that $a+m\left(b^{*}\right)=0$ ) is not an equilibrium. Either sponsor would gain by deviating and applying to a slightly less friendly SSO. The probability of endorsement would be slightly smaller but in case of endorsement, the standard would be perceived as slightly superior to the rival standard and therefore adopted for sure. ${ }^{33}$

\section{Proposition 18 Under standards competition,}

- the sponsors apply to less friendly (more credible) SSOs than they would if they were monopolists,

- the sponsors may apply to a single SSO or to multiple ones,

- equilibrium behavior is summarized in Figure 1.

\footnotetext{
${ }^{33}$ This reasoning is reminiscent of the "overtaking" arguments in Damiano-Li (2003) and BénabouTirole (2004).
} 


\section{Conclusion}

This paper examines firms' choices of intermediaries who can certify their property or ideas. While the problem arises in many aspects of economic life, we focus on the setting in which a firm is seeking the endorsement of a standard-setting organization for a proposed new technical standard.

The paper considers the choice between potential certifiers as a three-stage game: choice of certifier, endorsement, adoption. We highlight a number of conclusions:

- In a wide range of circumstances, sponsors will select only one SSO from which to receive certification, even when they are trying to get multiple sides on board.

- There is a negative relationship between the strength of proposed standard and credibility of the SSO selected by the sponsor to certify the standard.

- More extensive concessions to users will be made when the proposed standard is weaker.

- From a social welfare point of view, the sponsor of an attractive technology chooses an SSO that is too complacent and makes too few concessions in the design (unless she can perfectly capture the users' surplus through royalties). In case of a less attractive standard, regulation cannot improve on the sponsor's choices of SSO and design.

- An SSO that is more user-friendly than the sponsor would wish can engage in technology morphing. Its ability to do so, however, may be limited if the standard is only mildly attractive, as the required bargaining delays convey a negative signal about the standard.

- Independent users benefit when the sponsor has a stronger downstream presence.

- Standards competition induces sponsors to apply to more credible SSOs. 
This paper is, in many respects, only a first pass at modeling the choice and behavior of SSOs. One line of research would open the black box behind their preferences. In our model, the parameter $\alpha$ weighing the certifier's preferences over user and sponsor welfare was meant to reflect various dimensions of SSO governance, such as decisionmaking processes and composition of relevant committees, as well as their technological preferences. Another aspect worth investigating relates to the SSO's reputation-building process and the concomitant entry and exit dynamics in the SSO industry. Yet another topic of research would consist in introducing (endogenous) delays in SSO internal decision making and looking at their implications for the certification strategies of competing sponsors. Finally, while we have obtained some interesting results on both technology morphing and standards competition, our analysis only touches on the associated issues and ought to be extended in many directions such as the melding of multiple standards into a single one. We leave these topics and others for future research. 


\section{References}

[1] Admati, A.R., and P. Pfleiderer (1994) "Robust Financial Contracting and the Role of Venture Capitalists," Journal of Finance, 49: 371-402.

[2] Aghion, P., and J. Tirole (1997) "Formal and Real Authority in Organizations," Journal of Political Economy, 105: 1-29.

[3] An, M. (1998) "Logconcavity versus Logconvexity: A Complete Characterization," Journal of Economic Theory, 80: 350-369.

[4] Benabou, R., and J. Tirole (2004) "Incentives and Prosocial Behavior," mimeo, Princeton and IDEI.

[5] Besen, S.M., and L. L. Johnson (1986) Compatibility Standards, Competition and Innovation in the Broadcast Industry, Santa Monica, CA, The RAND Corporation.

[6] Caillaud, B., and J. Tirole (1999) "Party Governance and Ideological Bias," European Economic Review, 43: 779-790.

[7] Chemmanur, T.J., and P. Fulghieri (1994) "Investment Bank Reputation, Information Production, and Financial Intermediation," Journal of Finance, 49: 57-79.

[8] Damiano, E., and H. Li (2003) "Competing Match Making," mimeo, University of Toronto.

[9] Dessein, W. (2002) "Authority and Communication in Organizations," Review of Economic Studies, 69: 811-838.

[10] Diamond, D. (1984) "Financial Intermediation and Delegated Monitoring," Review of Economic Studies, 51: 393-414.

[11] Farrell, J. (1996) "Choosing the Rules for Formal Standardization," mimeo, University of California, Berkeley. 
[12] Farrell, J., and G. Saloner (1985) "Standardization, Compatibility, and Innovation," Rand Journal of Economics, 16: 70-83.

[13] — (1988) "Coordination Through Committees and Markets," Rand Journal of Economics, 19: 235-252.

[14] Farrell, J., and C. Shapiro (1992) "Standard Setting in High-Definition Television," Brookings Papers on Economic Activity: Microeconomics, 1-93.

[15] Lizzeri, A. (1999) "Information Revelation and Certification Intermediaries," Rand Journal of Economics, 30, 214-231.

[16] Meyer, M., and M. Rothschild (2004) "(In)efficiency in Sorting and Matching," seminar presentation, May 25, 2004, University of Toulouse.

[17] Morrison, A., and L. White (2004) "Financial Liberalization and Capital Regulation in Open Economies," mimeo, Oxford University and Harvard University.

[18] Peyrache, E., and L. Quesada (2003) "Strategic Certification," mimeo, HEC and University of Wisconsin.

[19] Rochet, J.C., and J. Tirole (2003) "Platform Competition in Two-Sided Markets," Journal of the European Economic Association, vol 1(4): 990-1029.

[20] Simcoe, T. (2003) "Committees and the Creation of Technical Standards," mimeo, University of California at Berkeley.

[21] Sirbu, M., and L. Zwimpfer (1985) "Computer Communications Standards: The Case of X.25," IEEE Communications, 23: 35-45.

[22] Weiss, M.B.H., and M. Sirbu (1990) "Technological Choice in Voluntary Standards Committees: An Empirical Analysis," Economics of Innovation and New Technology, 1: $111-133$. 